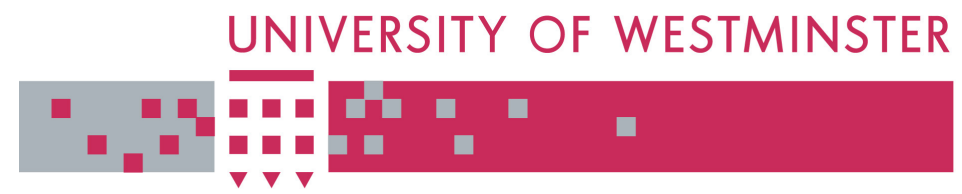

WestminsterResearch

http://www.wmin.ac.uk/westminsterresearch

\title{
A random effects sensitivity analysis for patient pathways model
}

Shola Adeyemi

Thierry J. Chaussalet

School of Informatics, University of Westminster

Copyright (C [2008] IEEE. Reprinted from the proceedings of the 21st IEEE International Symposium on Computer-Based Medical Systems, IEEE CBMS 2008, Jyväskylä, 17-19 June 2008. IEEE, Los Alamitos, USA, pp. 536-538. ISBN 9780769531656.

This material is posted here with permission of the IEEE. Such permission of the IEEE does not in any way imply IEEE endorsement of any of the University of Westminster's products or services. Personal use of this material is permitted. However, permission to reprint/republish this material for advertising or promotional purposes or for creating new collective works for resale or redistribution to servers or lists, or to reuse any copyrighted component of this work in other works must be obtained from the IEEE. By choosing to view this document, you agree to all provisions of the copyright laws protecting it.

The WestminsterResearch online digital archive at the University of Westminster aims to make the research output of the University available to a wider audience. Copyright and Moral Rights remain with the authors and/or copyright owners.

Users are permitted to download and/or print one copy for non-commercial private study or research. Further distribution and any use of material from within this archive for profit-making enterprises or for commercial gain is strictly forbidden.

Whilst further distribution of specific materials from within this archive is forbidden, you may freely distribute the URL of the University of Westminster Eprints (http://www.wmin.ac.uk/westminsterresearch).

In case of abuse or copyright appearing without permission e-mail wattsn@wmin.ac.uk. 


\title{
A Random Effects Sensitivity Analysis for Patient Pathways Model
}

\author{
Shola Adeyemi and Thierry J. Chaussalet \\ Health and Social Care Modelling Group \\ University of Westminster, London, United Kingdom \\ S.Adeyemi3,chausst(@wmin.ac.uk)
}

\begin{abstract}
In this paper, we present a random effects approach to modelling of patient pathways with an application to the neonatal unit of a large metropolitan hospital. This approach could be used to identify pathways such as those resulting in high probabilities of death/survival, and to estimate cost of care or length of stay. Patient-specific discharge probabilities could also be predicted as a function of the random effect. We also investigate the sensitivity of our modelling results to random effects distribution assumptions.
\end{abstract}

\section{Introduction}

One of the key elements in improving efficiency in health care services delivery is patient flow. Clinically, patient flow represents the progression of a patient's health status. Therefore, a good understanding of patient flow can offer knowledge and insight to health care providers, managers, administrators, and patients about the health care needs associated with medical concerns like disease progression or recovery status. Also, a good understanding of patient flow is also needed to support a health care facility's operational activities. From an operational perspective, patient flow can be thought of as the movement of patients through a set of locations in a health care facility. Effective resource allocation and capacity planning are contingent upon patient flow because patient flow, in the aggregate, is a good measure of demand for health care services [1]. Modelling patients flow in health care systems is considered to be vital in understanding the operational and clinical functions of the system and has proved to be useful in improving the functionality of the health care system. There has been a great deal of work on modelling patients flow in healthcare system. These works have used different techniques to model patient flow in health care systems with several different assumptions imposed on the system. Markov, compartmental models and Coxian Phase-type distributions have been used extensively to capture probabilistic laws that govern the dynamics of patients between states in the healthcare system $[2,3,4]$.

Modelling patient pathways was initiated with the work in [5] in which a semi Markov model was proposed for an individual patient's experience during a visit to a doctor's office. The stochastic model is based on tracking the visit of patients at a local family practice clinic. Also [6] developed a Markov models approach to show how patients' pathways may be extracted and describe an algorithm based on branch and bound developed to efficiently extract some interesting pathways. Though the work of previous researchers on patient pathways modelling are novel, short comings of their approaches include the fact that these are based on virtual states rather than physical states [6], inference are based on the population rather than on individual patient and patient history through the system is not incorporated hence they do not really model individual patient's experience in the process of care and discharge is to absorption (death). To deal with the shortcomings of these methods we initiate and develop a random effect modelling framework for patient pathways through an artificial system [7]. This approach could predict the probability of discharge from the system, as well as identify interesting pathways.

In this paper, we have proposed a random effect model for patient pathways with specific application to the neonatal system. We also investigate effect of different random effects distributions assumptions on the result of our model.

\section{Context}

We introduce, in this section, the neonatal system since the data used in this work concerns the neonatal unit of the University College of London Neonatal Unit.

\subsection{Neonatal System}

In the neonatal care unit, infants are taken into one of three units depending on some characteristics.

The Special Care Unit (SCU) provides a basic level of newborn care to infants at low risk. They have the capabilities to perform neonatal resuscitation at every delivery and to evaluate and provide routine postnatal care of healthy newborn infants

The High Dependency Unit (HDU) can provide care to infants who are ill with problems that are expected to resolve rapidly. 
The Intensive Care Unit (ICU) is defined as having continuously available personnel (neonatologists, neonatal nurses, respiratory therapists) and equipment to provide life support for as long as needed. This system is depicted in the figure below. Discharge from each unit could be by death, transfer to other hospitals, or home.

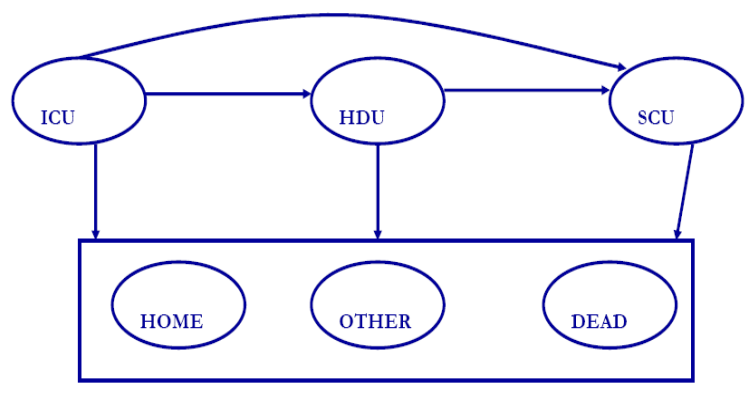

Figure 1: The Neonatal System

A restriction imposed on this system is that there is no backward flow of babies, though this is possible clinically.

\subsection{Data}

The data used is a UCLH, Neonatal Unit dataset of 1002 neonates admitted into the Neonatal Unit during the year 2006. There are 264 neonates admitted into the ICU, 93 to the HDU and 884 to the SCU. 102 neonates use facilities in the ICU only, 1 HDU only and 681 SCU only. There are 2 neonates that use facilities in ICU and HDU only, 43 in HDU and SCU only while we have 113 neonates using facilities in the ICU and SCU only. 47 neonates use facilities in ICU, HDU and SCU. There are 3 neonates we do not have information about and these are dropped from our analyses. In this paper, we propose a random effects model for patient pathways and apply same to the neonatal data as well as investigate the sensitivity of the model to different random effects distribution assumptions. In most studies, random effects are taken to be normally distributed, an assumption often made both for their appropriateness and for mathematical convenience. We extend the application of [8] to our multinomial logit model developed. In what follows, we briefly describe our patient pathways model.

\section{Methods and Application}

In this section, we introduce our proposed random effects pathways model.

\subsection{The Proposed Pathways Model}

Denote by $y_{p}=\left\{y_{p \mathbf{1}}, y_{p \mathbf{2}}, \ldots . ., y_{p n_{i}}\right\}$ the vector of response variables for the $p$ th patient, $p=1,2, \ldots, n$. The components or elements of $y_{p}$ could be continuous, binary or (censored) failure times. However, in our case, these are categorical outcomes. Outcomes in the same unit induce some correlation due to various shared features, introduced by a shared random effect $\theta_{p}$ for the $p$-th patient. The probability of passing through each state, via any of the pathway is modelled as

$$
P\left(y_{p i} \mid \beta, \theta_{p}\right)=\frac{\exp \left(X_{p} \beta+Z_{p} \theta_{p}\right)}{\sum \exp \left(X_{p} \beta+Z_{p} \theta_{p}\right)} ; y_{p i}=\mathbf{0}, \mathbf{1} \ldots, m_{i}-\mathbf{1},
$$

This is a random effects multinomial pathways model proposed in [7]. We assume that the random effects, $\theta_{p}$ has a density $f\left(\theta_{p} \mid \psi\right)$, with parameter $\psi, X_{p}, Z_{p}$ are the fixed and random effects design matrices respectively and $\beta$ is the fixed effects parameter. In most of these studies, random effects distribution $f\left(\theta_{p} \mid \psi\right)$ are taken to be normally distributed, an assumption often made both for their appropriateness and for mathematical convenience. As a result, most available user-friendly softwares focus on normal random effects models. However, results could be improved with appropriate random effects distribution assumptions. In this paper, to assess the sensitivity of inferences to assumptions about the distribution of the random effects $\theta_{p}$, we assume: (1) $\theta_{p}$ is normal with mean 0 and variance $\sigma^{2}$, (2) $\theta_{p}$ is gamma, (3) $\theta_{p}$ is exponential with mean $\lambda$, (4) $\theta_{p}$ is lognormal parameters $\mu=0$ and $\sigma^{2}=1$ and (5) $\theta_{p}$ is uniform on the unit interval.

\subsection{Application}

We apply a computational method based on probability integral transform as developed in [8] to obtain the maximum likelihood estimates (MLEs) in non-linear random effects models where the random effects are nonnormally distributed. The MLEs are obtained from the marginal likelihood, integrating over the random effects. Because the model is non-linear in the random effects, the marginal likelihood has no closed form, and a numerical integration approximation must be used. We have applied the method developed which is based on probability integral transform in which probability integral transform is used to transform a normal random effect to a nonnormal random effect. For all these we have applied the method using the adaptive Gaussian quadrature and implemented in SAS PROC NMLIXED.

We have presented, in Table 1, parameter estimates and standard errors of our model for different random effects distributions assumptions. We have also presented fit statistics; -2loglikelihood, Akaike Information Criterion (AIC) and Bayesian Information Criterion (BIC) to assess goodness of fit for the models. The results suggest that significance of the fixed effects parameters is not sensitive to the choice of the random effects distribution except in result based on exponential random effects. 


\begin{tabular}{|c|c|c|c|c|c|c|c|c|c|c|}
\hline \multicolumn{11}{|c|}{ Parameter Estimates } \\
\hline & \multicolumn{2}{|c|}{$\begin{array}{l}\text { Normal with } \\
\text { variance }=0.0003(0.0 \\
037)\end{array}$} & \multicolumn{2}{|c|}{$\begin{array}{l}\text { Exponential with } \\
\text { Lambda }=2.8249(0.4702)\end{array}$} & \multicolumn{2}{|c|}{$\begin{array}{l}\text { Uniform on the unit } \\
\text { interval }\end{array}$} & \multicolumn{2}{|c|}{ Lognormal } & \multicolumn{2}{|c|}{$\begin{array}{l}\text { Gamma, } \\
\text { lambda }=0.00005 \\
(0.000094)\end{array}$} \\
\hline Parameter & Estimate & SE & Estimate & $\mathrm{SE}$ & Estimate & SE & Estimate & SE & Estimate & SE \\
\hline $\mathrm{ICU}-\mathrm{OH}$ & 2.9140 & 0.5016 & 0.6075 & 0.6284 & 2.2515 & 0.5021 & 1.9356 & 0.5051 & 1.9140 & 0.5017 \\
\hline $\mathrm{HDU}-\mathrm{OH}$ & 0.8033 & 1.1693 & 0.3129 & 1.1740 & 0.6080 & 1.1761 & 0.4727 & 1.1756 & 0.4713 & 1.1750 \\
\hline $\mathrm{SCU}-\mathrm{OH}$ & 0.8260 & 0.1021 & 0.4707 & 0.1032 & 0.6922 & 0.1022 & 0.5879 & 0.1023 & 0.5893 & 0.1021 \\
\hline $\mathrm{ICU}-\mathrm{HDU}-\mathrm{OH}$ & -1.1946 & 1.2792 & -0.7002 & 1.2836 & -0.9978 & 1.2854 & -0.8622 & 1.2850 & -0.8612 & 1.2844 \\
\hline $\mathrm{ICU}-\mathrm{SCU}-\mathrm{OH}$ & -1.6229 & 0.4372 & -1.2782 & 0.4384 & -1.4885 & 0.4372 & -1.3855 & 0.4374 & -1.3859 & 0.4372 \\
\hline $\mathrm{HDU}-\mathrm{SCU}-\mathrm{OH}$ & -0.0758 & 1.1960 & 0.1977 & 1.2053 & 0.0529 & 1.2041 & 0.1265 & 1.2049 & 0.1231 & 1.2046 \\
\hline $\mathrm{ICU}-\mathrm{HDU}-\mathrm{SCU}-\mathrm{OH}$ & 0.8139 & 1.3684 & 0.5509 & 1.3773 & 0.6840 & 1.3755 & 0.6117 & 1.3762 & 0.6145 & 1.3759 \\
\hline ICU - HDU & 2.5722 & 0.4582 & 0.2586 & 0.5990 & 2.1099 & 0.4588 & 1.5932 & 0.4626 & 1.5724 & 0.4582 \\
\hline $\mathrm{HDU}-\mathrm{SCU}$ & 1.2572 & 0.4229 & 0.6960 & 0.4259 & 1.0601 & 0.4234 & 0.8973 & 0.4246 & 0.8945 & 0.4232 \\
\hline ICU - HDU - SCU & -1.0531 & 0.5541 & -0.4810 & 0.5588 & -0.8551 & 0.5545 & -0.6912 & 0.5557 & -0.6895 & 0.5544 \\
\hline $\mathrm{ICU}-\mathrm{SCU}$ & 1.2538 & 0.4630 & -1.0548 & 0.6002 & 0.7917 & 0.4636 & 0.2754 & 0.4672 & 0.2540 & 0.4631 \\
\hline ICU - HOME & 3.8281 & 0.4558 & 1.5225 & 0.5921 & 3.3658 & 0.4563 & 2.8499 & 0.4597 & 2.8280 & 0.4559 \\
\hline SCU - HOME & 1.8884 & 0.0809 & 1.5332 & 0.0833 & 1.7546 & 0.0810 & 1.6504 & 0.0812 & 1.6518 & 0.0809 \\
\hline SCU - HOME & -2.4067 & 0.3298 & -2.0635 & 0.3317 & -2.2730 & 0.3299 & -2.1701 & 0.3301 & -2.1703 & 0.3298 \\
\hline HDU - SCU - HOME & 0.3305 & 0.3942 & 0.1131 & 0.3982 & 0.2634 & 0.3953 & 0.2017 & 0.3971 & 0.1967 & 0.3955 \\
\hline ICU - HDU - SCU - HOME & -0.4654 & 0.5600 & -0.2332 & 0.5662 & -0.3975 & 0.5609 & -0.3343 & 0.5625 & -0.3303 & 0.5609 \\
\hline \multicolumn{11}{|c|}{ Fit Statistics } \\
\hline -2 Loglikelihood & \multirow{3}{*}{\multicolumn{2}{|c|}{$\begin{array}{l}3341.0 \\
3375.0 \\
3458.3\end{array}$}} & \multirow{3}{*}{\multicolumn{2}{|c|}{$\begin{array}{l}3227.2 \\
3261.2 \\
3344.5\end{array}$}} & \multirow{3}{*}{\multicolumn{2}{|c|}{$\begin{array}{l}3289.9 \\
3321.9 \\
3400.2\end{array}$}} & \multirow{3}{*}{\multicolumn{2}{|c|}{$\begin{array}{l}3253.9 \\
3285.9 \\
3364.3\end{array}$}} & \multirow{3}{*}{\multicolumn{2}{|c|}{$\begin{array}{l}3253.5 \\
3287.5 \\
3370.8\end{array}$}} \\
\hline AIC & & & & & & & & & & \\
\hline $\mathrm{BIC}$ & & & & & & & & & & \\
\hline
\end{tabular}

\section{Table1: Estimation results}

The MLEs of parameters in all the models, except the model with exponential random effects, are identical especially in the conclusion derived from them. These models yield significant estimates for the pathways except in HDU - OH; ICU - HDU - OH; ICU - HDU - SCU $\mathrm{OH}$; HDU - SCU - OH; ICU - HDU - SCU; ICU - SCU; HDU - SCU - Home and ICU - HDU - SCU - Home. In the exponential random effects model ICU $-\mathrm{OH}$ is also not significant, which suggests that the random effects distribution assumptions may affect the fixed effects parameters.

In terms of model fit, all the models describe the activities within the neonatal system satisfactorily since the non-significant pathways are those with few or no flow however, the model with exponential random effects distribution seems the most plausible model, considering the fit statistics (smaller is better). An explanation could be that the random effects captures the severity such that as babies progress through the system there is a drop in the severity because they get better which can be described by an exponential function.

\section{Conclusion}

We have shown that fixed effects parameters, sometimes, could be affected by random effects distribution assumptions. This is evident from the model with exponential random effects. The normal random effects model is out performed by all other models, which implies that assuming normal random effects raise many questions in practical applications of random effects modelling. The most plausible model is the one with exponential random effects. This study has also extended the application of the probability integral transform [8] to a multinomial logit random effects model. The pathways model proposed here could be applied in any context, though we have demonstrated its use in with a UCLH neonatal system however it can be used in other contexts where progressions are physical rather than virtual.

Acknowledgement: We are grateful to Dr Nikki Robbertson and $\mathrm{Mr}$ Daniel Wood for making the data used in this paper available and for useful discussion during the interpretation of our results.

\section{References}

[1] M.J. Cotes (2000). Understanding patients Flow, Production and Operations Management, Decision Line March, $8-10$ [2] V. Irvine, S. Mcclean, and P. Millard, "Stochastic-Models for Geriatric Inpatient Behavior," IMA Journal of Mathematics Applied in Medicine and Biology, vol. 11, pp. 207-216, 1994. [3] H. Xie, T. J. Chaussalet, and R. H. Millard, "A continuous time Markov model for the length of stay of elderly people in institutional long-term care," Journal of the Royal Statistical Society Series a-Statistics in Society, vol. 168, pp. 51-61, 2005. [4] M. J. Faddy and S. I. McClean, "Analysing data on lengths of stay of hospital patients using phase-type distributions," Applied Stochastic Models in Business and Industry, vol. 15, pp. 311-317, 1999.

[5] M. J. Cote and W. E. Stein, "A stochastic model for a visit to the doctor's office," Mathematical and Computer Modelling, vol. 45, pp.309-323, 2007.

[6] S. McClean, 1. Garg, B. Meenan and P. H. Millard, "Using Markov Models to find Interesting Patient Pathways," Proc. 20th IEEE Symp. CBMS, pp. 713-718, 2007.

[7] S. Adeyemi, T. J. Chaussalet, H. Xie and P. H. Millard, "Patients flow: a mixed-effects modelling approach to predicting discharge probabilities," Proc. 20th IEEE Symp. CBMS, pp. 725-730, 2007.

[8] K.P. Nelson, S. Lipsitz, G.E. Fitzmaurice, J. Ibrahim, M. Parzen and R. Strawderman, "Use of Probability Integral Transformation to Fit Mixed-Effects Models With Nonnormal Random Effects", Journal of Computational and Graphical Statistics, vol. 15(1), pp. 39 - 57, 2006. 